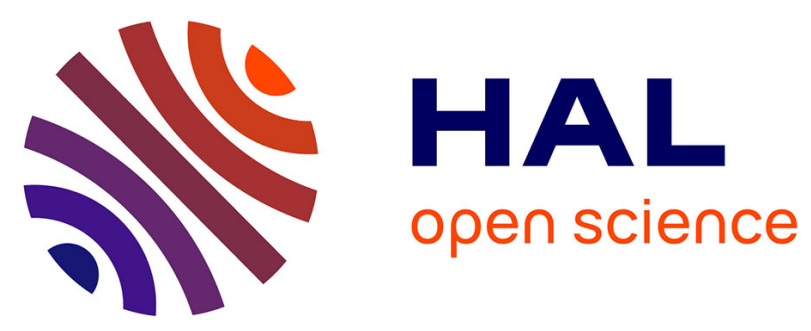

\title{
A Nonlinear Filtering Approach for the Attitude and Dynamic Body Acceleration Estimation Based on Inertial and Magnetic Sensors: Bio-Logging Application
}

Hassen Fourati, Noureddine Manamanni, Lissan Afilal, Yves Handrich

\section{- To cite this version:}

Hassen Fourati, Noureddine Manamanni, Lissan Afilal, Yves Handrich. A Nonlinear Filtering Approach for the Attitude and Dynamic Body Acceleration Estimation Based on Inertial and Magnetic Sensors: Bio-Logging Application. IEEE Sensors Journal, 2011, 11 (1), pp. 233-244. 10.1109/JSEN.2010.2053353 . hal-00624142

\section{HAL Id: hal-00624142 \\ https://hal.science/hal-00624142}

Submitted on 15 Sep 2011

HAL is a multi-disciplinary open access archive for the deposit and dissemination of scientific research documents, whether they are published or not. The documents may come from teaching and research institutions in France or abroad, or from public or private research centers.
L'archive ouverte pluridisciplinaire HAL, est destinée au dépôt et à la diffusion de documents scientifiques de niveau recherche, publiés ou non, émanant des établissements d'enseignement et de recherche français ou étrangers, des laboratoires publics ou privés. 


\title{
A Nonlinear Filtering Approach for the Attitude and Dynamic Body Acceleration Estimation Based on Inertial and Magnetic Sensors: Bio-Logging Application
}

\author{
Hassen Fourati, Student Member, IEEE, Noureddine Manamanni, Lissan Afilal, and Yves Handrich
}

\begin{abstract}
This paper addresses the problem of rigid body orientation and Dynamic Body Acceleration (DBA) estimation. This work is applied in bio-logging, an interdisciplinary research area at the intersection of animal behavior and bioengineering. The proposed approach combines a quaternion-based nonlinear filter with the Levenberg Marquardt Algorithm (LMA). The algorithm has a complementary structure design that exploits measurements from a three-axis accelerometer, a three-axis magnetometer, and a three-axis gyroscope. Attitude information is necessary to calculate the animal's DBA in order to evaluate its energy expenditure. Some numerical simulations illustrate the nonlinear filter performance. Some quantitative assessments prove this efficiency such as the time constant of the filter $(\tau=2 \mathrm{~s})$ and the $\mathrm{rms}$ magnitude of the quaternion error $(\mathrm{rms}=0.0156)$. Moreover, the effectiveness of the algorithm is experimentally demonstrated. In the experiments a domestic animal is equipped with an Inertial Measurement Unit (MTi-G), which provides a truth attitude for comparison with the complementary nonlinear filter. The rms difference between the filter and MTi-G outputs in the free movement experiments is within $0.392^{\circ} \mathrm{rms}$ on roll, $0.577^{\circ} \mathrm{rms}$ on pitch, and $2.521^{\circ} \mathrm{rms}$ on yaw.
\end{abstract}

Index Terms-Attitude, bio-logging, complementary nonlinear filter, dynamic body acceleration (DBA), inertial measurement unit (MTi-G), MEMS inertial/magnetic sensors.

\section{INTRODUCTION}

$\mathbf{N}$ OWADAYS, the attitude estimation of a moving rigid body is involved in several fields. For example, in aerial and marine vehicles [1]-[3], the attitude is used to achieve a stable controller. In rehabilitation and biomedical engineering [4], the orientation is necessary to record patient movements and providing adequate feedback for the therapist.

Manuscript received April 07, 2010; revised May 26, 2010; accepted May 26, 2010. Date of publication September 20, 2010; date of current version November 10, 2010. This work was supported in part by the Alsace and Champagne-Ardenne regions through Project NaviMeles. The associate editor coordinating the review of this manuscript and approving it for publication was Prof. Boris Stoeber.

H. Fourati, N. Manamanni, and L. Afilal are with CReSTIC-URCA, Reims Champagne Ardenne University, UFR SEN, 51687 Reims, Cedex 02, France (e-mail : hassen.fourati@univ-reims.fr; hassen.fourati@c-strasbourg.fr; noureddine.manamanni@univ-reims.fr; lissan.afilal@univ-reims.fr).

Y. Handrich is with IPHC-DEPE, UdS, CNRS (Institut Pluridisciplinaire $\mathrm{Hu}-$ bert CURIEN/Département Ecologie, Physiologie et Ethologie), 67087 Strasbourg, France (e-mail: yves-jean-handrich@c-strasbourg.fr).

Color versions of one or more of the figures in this paper are available online at http://ieeexplore.ieee.org.

Digital Object Identifier 10.1109/JSEN.2010.2053353
Recently, the problem of attitude and orientation tracking are treated in bio-logging. This scientific field involves both bioengineering and animal behavior and aims to obtain new information on the natural world, which provides new insights into the hidden lives of animal species [5], [6]. Generally, bio-logging involves attaching an electronic device to a free-ranging animal in order to record different aspects of the animal's biology [7] (e.g., 3-D movements, behavior, physiology, and energy expenditure) and environment.

The main goal of this paper is to suggest an option concerning a body motion estimation method [body attitude and dynamic body acceleration (DBA)]. The latter is suitably used for marine mammals or birds. Marine animals are particularly hard to study during their long foraging trips at sea. However, their need to return periodically to the breeding colony gives us the opportunity to measure these different parameters using bio-logger devices. Note that the use of inertial and magnetic sensors is relatively recent, due to the difficulty to develop miniaturized technologies adapted to high rate record sampling (over $12-50 \mathrm{~Hz}$ ). The obvious advantage of this new approach is that we gain access to the third dimension space, which is the key to a good understanding of the diving strategies observed in these predators [8], [9]. The principle, proposed in those papers for tracking the animal's posture estimates in the aforementioned studies, consists of using a three-axis accelerometer and a three-axis magnetometer, with the reductive assumption that all movements are static or quasi-static. This assumption made, we can consider that the accelerometer outputs correspond to the gravity measurements in body coordinates, and it therefore becomes possible to estimate the attitude by the resolution of well known Wahba's problem [8]. However, the assumption made above is not valid in all dynamic situations observed underwater in freeranging animals, e.g., the phases of prey pursuit. Thus, the performance of attitude estimation is significantly degraded. Other work as [10] is focused on the use of a low-pass filter to extract the gravity projection from accelerometer outputs. In our knowledge, the tune of this filter is not easy, which introduces some errors on the attitude in many cases. In [11], the authors assumed that the running means over a one second interval of total acceleration time is a good estimator of the gravity vector projection. However, this approximation may not be valid over time and depends on other parameters such as animal species and the type of movement it makes.

In this paper, we propose the addition of a three-axis gyroscope measurement to the sensors already used (accelerom- 
eter and magnetometer) in order to overcome the drawbacks of aforementioned papers in this area [8]-[11]. Indeed, the estimation of attitude using fusion technologies with low-cost sensors such as gyroscopes, accelerometers, and magnetometers has already been used in other fields. Micro Air Vehicles [1], [3] and biomedical engineering [4] are examples using either Kalman filters (KFs) [12], extended Kalman filters (EKFs) [13], or nonlinear observers [14]. It is important to note that the rigid body attitude estimation using MEMS inertial and magnetic sensors is useful for calculating the DBA of the moving body, although the proposed methodology based on the use of these sensors in not a new one. Nevertheless, we propose another way to combine these measurements and the developed approach still the first applied work, in our knowledge, in bio-logging.

The main idea of the algorithm is to use a complementary nonlinear filter coupled with a Levenberg Marquardt Algorithm (LMA) to process the measurements from a three-axis gyroscope, a three-axis magnetometer, and a three-axis accelerometer. The proposed approach combines a strap-down system, based on the time integral of the angular velocity, with the LMA that uses the earth's magnetic field and gravity vector to compensate the attitude predicted by the gyroscope. It is important to note that the resulting structure is complementary: high-bandwidth-rate gyro measurements are combined with low-bandwidth vector observations (gravity and earth's magnetic field) to provide an accurate attitude estimate. Furthermore, the estimated attitude is used to calculate three components of DBA, which provides important information about the energy budgets of free-living animals.

This paper is organized as follows. Section II describes the prototype design for the bio-logging application. Moreover, the attitude parameterization, the rigid body kinematic model, and sensor measurement models are presented. Section III details the structure of the proposed complementary nonlinear filter for the attitude and DBA estimation. Sections IV and V are devoted to simulation and experimental results to illustrate the effectiveness of the proposed algorithm. Finally, Section VI summarizes the main conclusions of the paper.

\section{MAterials AND MethodS}

The prototype "logger," the attitude representation, and sensor models are described here.

\section{A. Bio-Logging Application: Case Study and Logger Concept}

Bio-logging refers to the use of electronic devices (logger) attached to the animal. The King penguin is one of the major models of diving birds studied at Strasbourg University thanks to the bio-logging technology [7]. A future generation of loggers should be equipped with a kinematic sensing unit (ADIS16405 from Analog Device [15]) that integrates a three-axis accelerometer, a three-axis magnetometer, and a three-axis gyroscope. It is a micro-fabricated device that provides digital signals $\left( \pm 5 \mathrm{~g}, \pm 300^{\circ} / \mathrm{s}, \pm 2\right.$ Gauss, and $23 \mathrm{~mm} \times 23 \mathrm{~mm} \times 23 \mathrm{~mm}$ ). The core of the hardware would be an ATMEGA 644-QFN ultra low-power microcontroller [16] including several on-ship peripherals. It should read digital signals from the ADIS16405 via SPI bus. Other kinds of sensors need to be implemented in the prototype, such as a pressure transducer (Keller-3L from KELLER [17]) and a temperature sensor (TC77 from Microchip [18]) to obtain information about the animal's environment. These sensors have to be periodically sampled with different frequencies, filtered, corrected from errors and temperature effects, and stored for postprocessing. The main storage of data is provided by a flash memory (Micro SD from San Disk [19]). A prototype would be protected from the effect of low temperature and high-pressure data by a special material called resin [20] and powered by a 3.6-V lithium battery. To maximize battery life time, the system should be automatically started for periodically moments of the day. With these unit and sensors, the power consumption of the prototype is estimated around $350 \mathrm{~mW}$ of which most is consumed by the sensors. Once the bird returns from a foraging trip at sea, the logger should be recovered, and all recorded measurements on the memory are downloaded and used into the nonlinear filter. Note that the loggers are autonomous and do not use a satellite or RF transmission.

Before deploying such a logger, the goal in this paper is to be able to convert the raw data (acceleration, angular rate, and earth's magnetic field) into relevant information: attitude and energy expenditure (DBA). The algorithms that will exploit the measurements from this prototype are the main concerns of this work. It is important to note that during preliminary experimental tests in the paper, equivalent devices available on the market are used to evaluate the performance of the attitude estimation algorithm. Then, Inertial Measurement Units were used, namely, the MTi and MTi-G from Xsens technologies [21]. These units integrate the main sensors for attitude estimation such as a three-axis accelerometer, a three-axis magnetometer, and a three-axis gyroscope.

\section{B. Rigid Body Attitude Description}

The rigid body attitude in space is determined when the axis orientation of the body frame $B\left(X_{B}, Y_{B}, Z_{B}\right)$ is specified with respect to the navigation frame $N\left(X_{N}, Y_{N}, Z_{N}\right)$. The navigation frame is attached to the earth and tangent on its surface (N-E-D: $X_{N}$ : Magnetic North, $Y_{N}$ : East, $Z_{N}$ : Down) [22].

In this paper, we consider the unit quaternion as the mathematical representation of rigid body attitude between the mentioned frames. The unit quaternion, denoted by $g$, is a hypercomplex number of rank 4 such that

$$
q=\left[\begin{array}{ll}
s & v^{T}
\end{array}\right]^{T}
$$

where $s$ is the scalar part and $v=\left[\begin{array}{lll}v_{x} & v_{y} & v_{z}\end{array}\right]^{T}$ is the vector part of the quaternion. We invite the reader to refer to the Appendix and [23] for more details about quaternion algebra.

\section{Sensor Measurement Models}

The sensors configuration consists of a three-axis gyroscope, a three-axis accelerometer, and a three-axis magnetometer containing MEMS technologies [24], [25]. Their theoretical outputs can be written, respectively, as follows:

$$
\begin{aligned}
\omega_{G} & =\left[\begin{array}{lll}
\omega_{G x} & \omega_{G y} & \omega_{G z}
\end{array}\right]^{T}=\omega+b+\delta_{G} \\
f & =\left[\begin{array}{lll}
f_{x} & f_{y} & f_{z}
\end{array}\right]^{T}=M_{N}^{B}(q)(g+a)+\delta_{f} \\
h & =\left[\begin{array}{lll}
h_{x} & h_{y} & h_{z}
\end{array}\right]^{T}=M_{N}^{B}(q) m+\delta_{h}
\end{aligned}
$$


where $b \in \Re^{3}$ is the unknown gyro-bias vector and $\delta_{G}, \delta_{f}$, and $\delta_{h} \in \Re^{3}$ are assumed white Gaussian noises. $\omega$ represents the real angular velocity, $g$ is the gravity vector, $a$ denotes the DBA, and $m$ describes the direction of the earth's magnetic field. $M_{N}^{B}(q)$ is the rotation matrix defined in (18) (see the Appendix).

\section{Filter Design for AtTITUde AND DBA Estimation}

In this paper, the objective is to design a rigid body attitude estimation algorithm based on inertial and magnetic MEMS sensors. The proposed approach will subsequently be used to track the orientation and DBA during several motions in free-ranging animals. This work is proposed in order to improve the attitude estimation approaches developed in [8]-[11] and limited to the static and quasi-static cases of animal's movements. We propose an approach based on a nonlinear filter to gain the most accurate attitude estimation using the advantages of accelerometers, magnetometers and gyroscopes. It is important to note that the resulting approach structure is complementary: high-bandwidth-rate gyro measurements are combined with low-bandwidth vector observations to provide an accurate attitude estimate [26].

\section{A. Attitude Filter Description}

To achieve our goal, (20) is developed to form the nonlinear system ( $\Im$ ) given by (5), shown at the bottom of the page, where $q \in \Re^{4}$ is the state vector, $I_{3 \times 3}$ is the identity matrix, and $\left[v^{\times}\right]$ is defined in (17) (see the Appendix). The output $y \in \Re^{6}$ of this system is built by stacking the accelerometer and magnetometer measurements such as

$$
y=\left[\begin{array}{llllll}
f_{x} & f_{y} & f_{z} & h_{x} & h_{y} & h_{z}
\end{array}\right]^{T} .
$$

In order to estimate the attitude, the following nonlinear filter is proposed [based on (5) and (6)]:

$$
\begin{aligned}
& {\left[\begin{array}{c}
\dot{\hat{s}} \\
\hat{\hat{v}}_{x} \\
\hat{\hat{v}}_{y} \\
\dot{\hat{v}}_{z}
\end{array}\right]=\frac{1}{2}\left[\begin{array}{r}
-\hat{v}_{x} \omega_{x}-\hat{v}_{y} \omega_{y}-\hat{v}_{z} \omega_{z} \\
\hat{s} \omega_{x}-\hat{v}_{z} \omega_{y}+\hat{v}_{y} \omega_{z} \\
\hat{v}_{z} \omega_{x}+\hat{s}_{y} \omega_{y} \hat{v}_{x} \omega_{z} \\
\hat{v}_{x} \omega_{y}-\hat{v}_{y} \omega_{x}+\hat{s} \omega_{z}
\end{array}\right]} \\
& \qquad\left[\begin{array}{llllll}
1 & 0 & 0 & 0 & 0 & 0 \\
0 & & & \\
0 & & K & \\
0 & &
\end{array}\right]
\end{aligned}
$$

where $\hat{q}=\left[\begin{array}{llll}\hat{s} & \hat{v}_{x} & \hat{v}_{y} & \hat{v}_{z}\end{array}\right]^{T} \in \Re^{4}$ and $K \in \Re^{3 \times 6}$ denote the estimated states (attitude) and the filter gain, respectively. Note that $\delta(\hat{q})=(y-\hat{y})$ is the modeling error which represents the

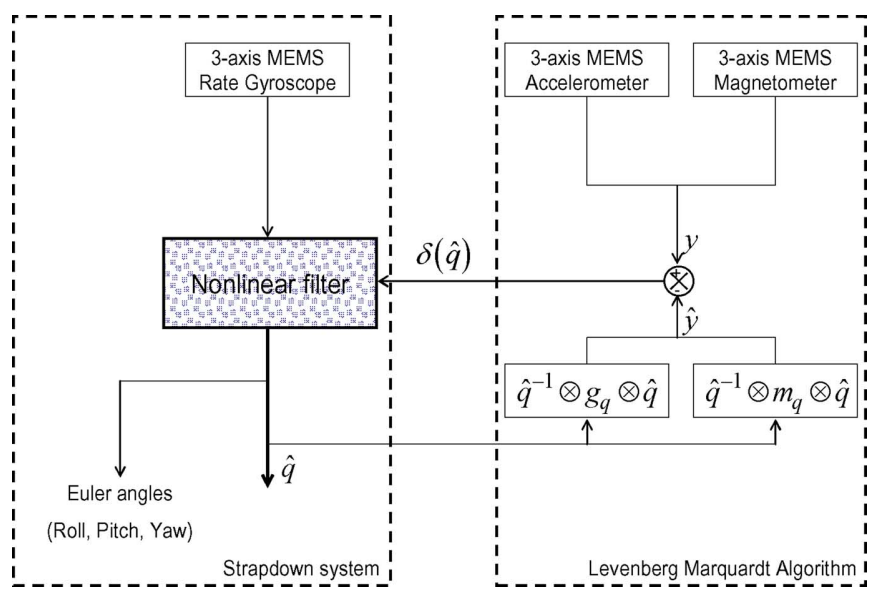

Fig. 1. Block diagram for the attitude estimation algorithm.

difference between real measurements $y$, as defined in (6), and estimated values $\hat{y}$, given by

$$
\hat{y}=\left[\begin{array}{llllll}
\hat{f}_{x} & \hat{f}_{y} & \hat{f}_{z} & \hat{h}_{x} & \hat{h}_{y} & \hat{h}_{z}
\end{array}\right]^{T} .
$$

The components of $\hat{y}$ are calculated using (21) and (22) (see the Appendix). The filter gain $K$ in (7) is used to correct the modeling error $\delta(\hat{q})$. This can be done if we are able to locate the minimum of the scalar squared error criterion function defined such as

$$
\xi(\hat{q})=\delta(\hat{q})^{T} \delta(\hat{q}) .
$$

In this paper, the LMA is used to minimize the nonlinear function $\xi(\hat{q})$ [27]. Then, the unique minimum can be written in the following form [28], [29]:

$$
\eta(\hat{q})=K \delta(\hat{q})=k\left[X^{T} X+\lambda I_{3 \times 3}\right]^{-1} X^{T} \delta(\hat{q})
$$

where $\lambda \in \Re$ guarantees that the inverted term will be nonsingular. $X \in \Re^{6 \times 3}$ is the Jacobian matrix defined in (23) (see the Appendix).

\section{B. Nonlinear Filter Performance Analysis}

The scheme of the attitude estimation algorithm is summarized in Fig. 1. A frequency analysis of accelerometer signals [see (3)] shows that gravity components $(g)$ tend to lie towards the low end of the frequency spectrum while the DBA $(a)$ has higher frequencies components [26]. The frequency analysis of magnetometer measurements shows also that the magnetic field tends to lie towards the low end of the frequency spectrum [see (4)] [26]. The gyroscope signals analysis shows that the drift $b$, which is a slow-moving process [see (2)], tends to lie towards

$$
(\Im):\left\{\dot{q}=\left[\begin{array}{c}
\dot{s}^{s} \\
\dot{v}_{x} \\
\dot{v}_{y} \\
\dot{v}_{z}
\end{array}\right]=\frac{1}{2}\left[\begin{array}{c}
s \\
v_{x} \\
v_{y} \\
v_{z}
\end{array}\right] \otimes\left[\begin{array}{c}
0 \\
\omega_{x} \\
\omega_{y} \\
\omega_{z}
\end{array}\right]=\frac{1}{2}\left[\begin{array}{c}
-v^{T} \\
I_{3 \times 3} s+\left[v^{\times}\right]
\end{array}\right]\left[\begin{array}{c}
\omega_{x} \\
\omega_{y} \\
\omega_{z}
\end{array}\right]\right.
$$


the low end of the spectrum, and the angular velocity $\omega$ tends to have higher frequency components [26]. Therefore, signals coming from the accelerometer-magnetometer pair and signals from the gyroscope have a complementary frequency spectrum [26]. The resulting structure of the nonlinear filter is complementary: it blends the low-frequency region (low bandwidth) of the accelerometer and magnetometer data, where the attitude is typically more accurate, with the high-frequency region (high bandwidth) of the gyroscope data, where the integration of the angular velocity yields better attitude estimates. Indeed, the filtering approach can perform a low-pass filtering on the signals from the accelerometer-magnetometer pair and a high-pass filtering on the signals from the rate gyroscope. By filtering the high-frequency components of the signals from the accelerometer (DBA) and the low-frequency components of the gyroscope signal (slow-moving drift), the nonlinear filter produces an accurate estimate of the attitude. More details on the filter performance are given in the Appendix.

\section{Simulation Results}

This section aims to illustrate the performance and accuracy of the designed complementary nonlinear filter. Some numerical simulations were carried out under MATLAB to estimate a rigid body attitude based on theoretical inertial and magnetic measurements. We consider an attitude variation example taken from angular velocity data over $50 \mathrm{~s}$. The following angular rate values are simulated:

$$
\begin{aligned}
& \text { for } \mathrm{t} \leq 25 \mathrm{~s} \\
& \left\{\begin{array} { l } 
{ \omega _ { x } ( t ) = - 1 . 8 \operatorname { s i n } ( 1 . 5 \mathrm { t } ) } \\
{ \omega _ { y } ( t ) = 0 . 5 \operatorname { c o s } ( 0 . 9 \mathrm { t } ) } \\
{ \omega _ { z } ( t ) = 1 . 5 \operatorname { s i n } ( 1 . 2 \mathrm { t } ) }
\end{array} \quad \left\{\begin{array}{l}
\omega_{x}(t)=-0.9 \sin (\mathrm{t}) \\
\omega_{y}(t)=\cos (-0.9 \mathrm{t}) . \\
\omega_{z}(t)=0.9 \sin (2 \mathrm{t})
\end{array}\right.\right.
\end{aligned}
$$

Then, the kinematic equation (5) is solved to obtain the continuous time motion in quaternion representation using the angular velocity measurements in (11). The obtained quaternion is used as a reference to compare it with the estimated quaternion from the proposed nonlinear filter. Moreover, the specific force and magnetic field measurements are created using (3) and (4). The rotation matrix $M_{N}^{B}(q)$ in (18) (see the Appendix) is computed using the theoretical quaternion (reference). To represent the sensor imperfections, an additive random zero-mean white Gaussian noise and disturbance was considered for all measurements, with a large standard deviation (see Table I). The angular velocity measurements are also assumed to be corrupted by theoretical bias given by

$$
b=\frac{\pi}{180}\left[\begin{array}{lll}
-5.2 & 6 & 4.3
\end{array}\right]^{T}\left(\frac{\mathrm{rad}}{\mathrm{s}}\right) .
$$

Note that the bias is very common and is an undesirable characteristic of low-cost rate gyros. The sampling rate was chosen as $100 \mathrm{~Hz}$ for all measurements.

The theoretical components of the quaternion (reference motion) as well as those of the nonlinear filter are initialized with different random values. These conditions are summarized in Table II. Notice that this choice allows us to illustrate the convergence of the filter even though it was initialized far from the actual states. At each integration step, (5) is resolved to obtain the theoretical quaternion $q$. Thereafter, accelerometer and magne-
TABLE I

CHARACTERISTICS OF THE VARIOUS NOISES FOR SENSOR MEASUREMENTS

\begin{tabular}{cccc}
\hline \hline Sensors & Parameters & $\begin{array}{c}\text { Standard } \\
\text { deviations }\end{array}$ & Units \\
\hline Accelerometer & $\delta_{f}$ & 0.1 & $\mathrm{~m} / \mathrm{s}^{2}$ \\
Magnetometer & $\delta_{h}$ & 0.1 & $\mathrm{Gauss}$ \\
Gyroscope & $\delta_{G}$ & 0.2 & $\mathrm{rad} / \mathrm{s}$ \\
\hline \hline
\end{tabular}

TABLE II

INITIAL CONDITIONS

\begin{tabular}{ll}
\hline \hline \multicolumn{3}{c}{ Quaternion } \\
\hline $\begin{array}{c}\text { Theoretical } \\
\text { model }\end{array}$ & $q\left(t_{0}\right)=\left[\begin{array}{llll}1 & 0 & 0 & 0\end{array}\right]^{T}$ \\
$\begin{array}{c}\text { Nonlinear } \\
\text { filter }\end{array}$ & $\hat{q}\left(t_{0}\right)=\left[\begin{array}{llll}0.1 & 0.9 & 1 & 0.7\end{array}\right]^{T}$ \\
\hline \hline
\end{tabular}

tometer measurements are used in the LMA to calculate the correction term $\Delta$ (see (24) and (25) in the Appendix). Finally, (7) is solved to obtain the estimated quaternion $\hat{q}$. The filter gain $k$, from (10), guarantees convergent estimates and is set according to the considered sensor noise levels as $k=5$. The constant $\lambda$ in (10) is chosen as $\lambda=10^{-6}$. Notice that the integration loop of the algorithm has the length $i=50 / 0.01=5000$.

Fig. 2 shows the flowchart representation of the estimation algorithm. It gives a step-by-step solution to the given problem (attitude estimation) using a simulation experiment.

The time histories of the quaternion $q$ (reference) as well as filter estimates $\hat{q}$ are shown in Fig. 3. Despite the fact that the nonlinear filter and theoretical model of quaternion were initialized with different initial conditions, one can note that the estimated quaternion rapidly converges towards the theoretical values. The filter converges quite fast with a time constant $\tau=2$ sec. It is important to note that the rate gyro bias and noises in the accelerometer and magnetometer measurements are rejected by the filter. In order to evaluate the overall performance of the attitude estimation, we plotted the time history evolution of the quaternion estimation error. This error is computed using (19) (see the Appendix). Fig. 4 depicts the convergence of this error towards zero during the simulated motion. Moreover, the filter performance is shown quantitatively using the root mean square error (rmse) in (31) (see the Appendix). So, the orientation estimates are accurate within 0.0156 RMS on the estimation error (19). In conclusion, the obtained results show that the complementary nonlinear filter is an efficient method to improve the quality of sensor measurements and attitude estimation.

\section{EXPERIMENTAL RESULTS AND DISCUSSION}

\section{A. Experimental Setup}

In order to evaluate the efficiency of the proposed approach in real-world applications, an experimental setup was developed. Two inertial measurement units were used, namely the MTi and MTi-G from Xsens Technologies [21], which output data at a rate of $100 \mathrm{~Hz}$. The Xsens devices are miniature, lightweight, and 3-D digital output sensors. They output a 3-D acceleration from accelerometer, a 3-D angular rate from gyroscope, and a 3-D magnetic field data from magnetometer with a 


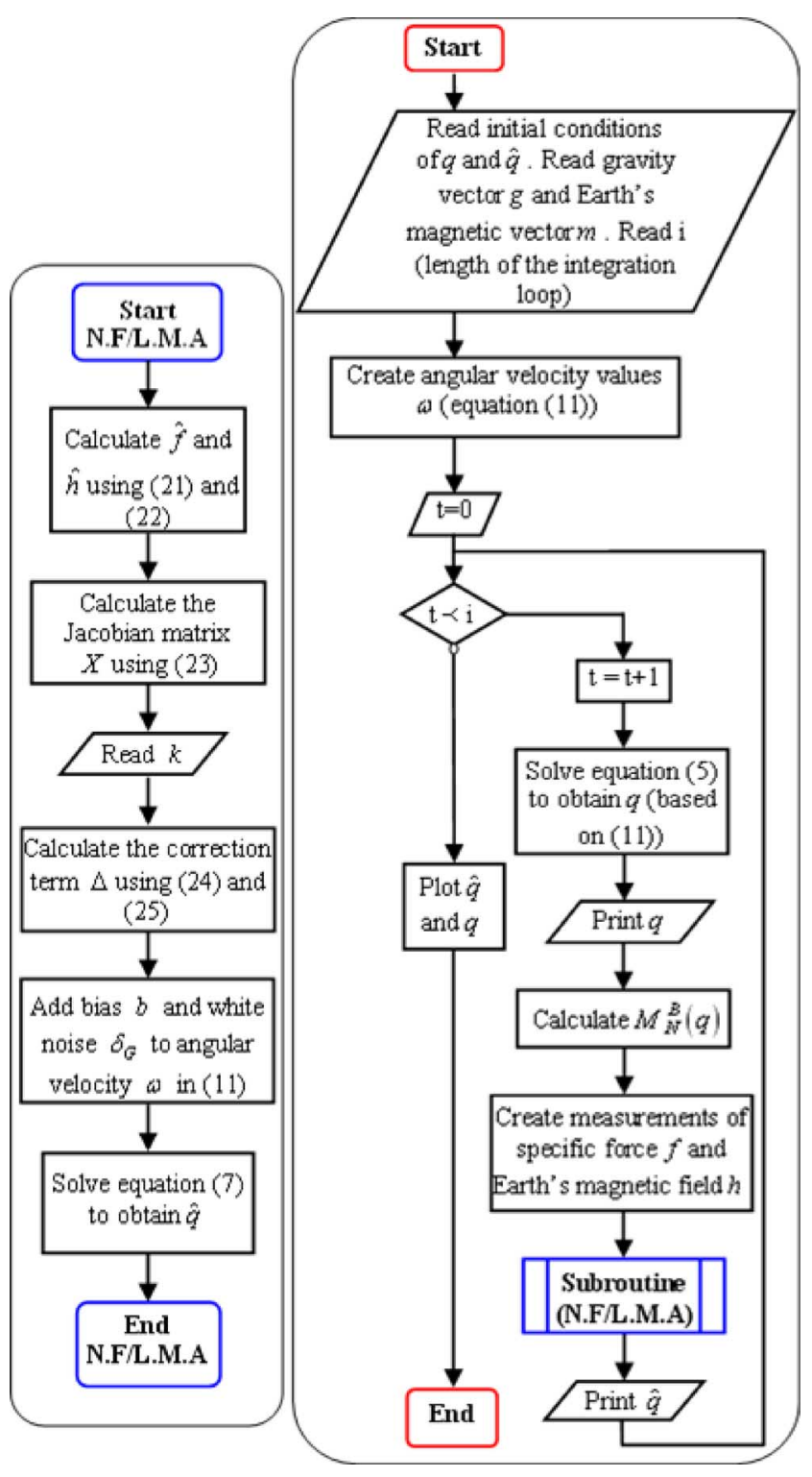

Fig. 2. Flowchart representing the estimation algorithm.

built-in bias, sensitivity, and temperature compensation. Note that the MTi-G is a GPS-enhanced attitude and heading reference system (AHRS). The Xsens MT devices can track the attitude in Euler angles, quaternion, and rotation matrix representations. Table III summarizes the sensor specifications of the Xsens MT devices (see the Appendix). It is important to note that MT devices serve as tools for the evaluation of the nonlinear filter efficiency and cannot be suitable for use in free-ranging animals due to their dependences on an energy source as well as their heavy weights. In the following set of experiments, the calibrated data from the MTi and MTi-G are used as inputs to the proposed filter.

\section{B. Attitude Estimation by the MTi and MTi-G}

The orientation determined by the MTi is computed using a KF. It uses the gravity and earth's magnetic field in order to compensate the slowly increasing drift from the time integral of angular velocity. The KF uses the assumption that on average
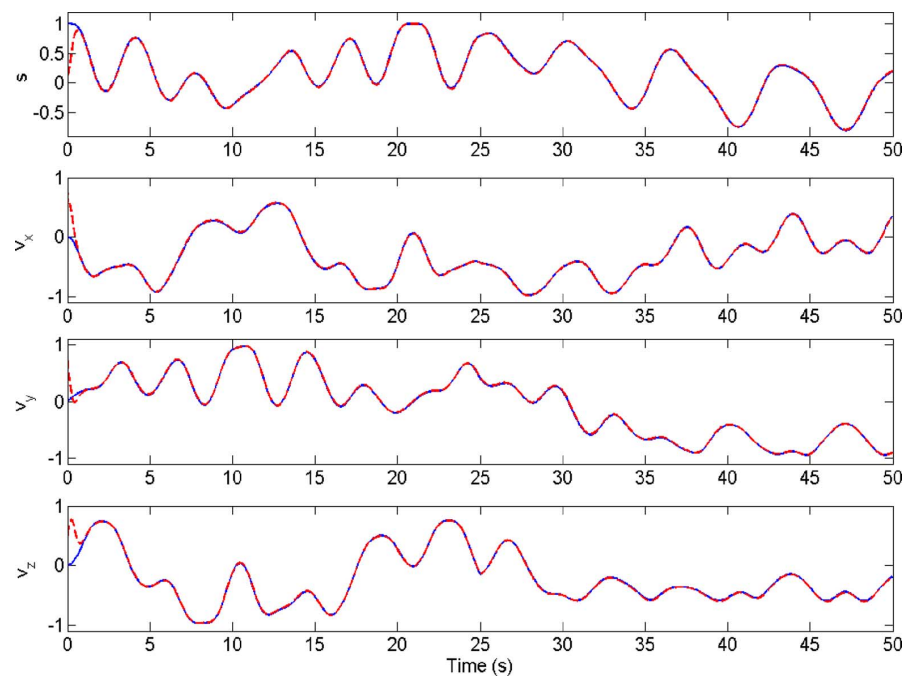

Fig. 3. Four components of the quaternion: Theoretical model (solid line) and estimated quaternion from the nonlinear filter (dashed line).

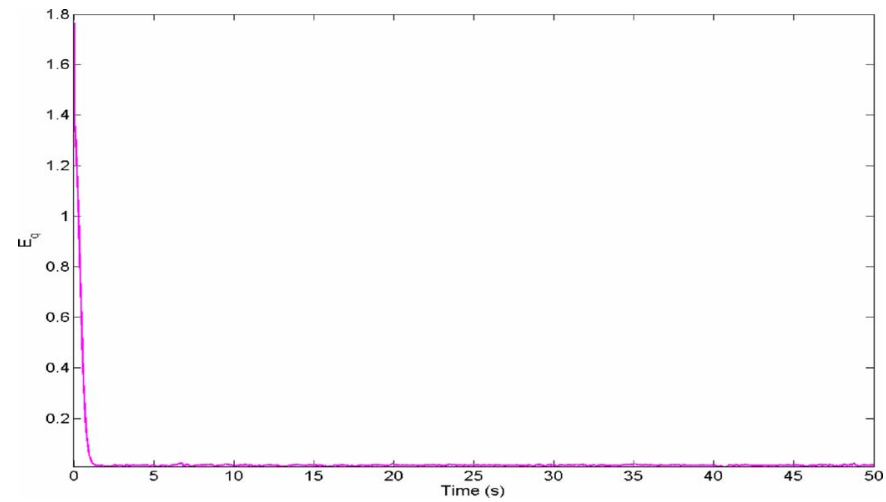

Fig. 4. Estimation error $E_{q}=\left\|\left(q^{-1} \otimes \hat{q}\right)-1\right\|$.

TABLE III

CAlibrated Data Performance SPECification

\begin{tabular}{cccc}
\hline \hline $\begin{array}{c}\text { Sensor } \\
\text { performance }\end{array}$ & Rate of turn & Acceleration & $\begin{array}{c}\text { Magnetic } \\
\text { field }\end{array}$ \\
\hline Full scale & $\pm 300 \mathrm{deg} / \mathrm{s}$ & $\pm 50 \mathrm{~m} / \mathrm{s}^{2}$ & $\pm 750 \mathrm{mGauss}$ \\
Bias stability & $1 \mathrm{deg} / \mathrm{s}$ & $0.02 \mathrm{~m} / \mathrm{s}^{2}$ & $0.1 \mathrm{mGauss}$ \\
$\begin{array}{c}\text { Noise } \\
\text { Alignment } \\
\text { error }\end{array}$ & $0.05 \mathrm{deg} / \mathrm{s} / \sqrt{\mathrm{Hz}}$ & $0.002 \mathrm{~m} / \mathrm{s}^{2} / \sqrt{\mathrm{Hz}}$ & $0.5 \mathrm{mGauss}$ \\
\hline \hline
\end{tabular}

the acceleration due to the movement (linear acceleration) is zero [21]. Then, during the time of this assumption, the gravity direction can be estimated and used to stabilize the attitude obtained from the gyroscope (roll and pitch angles). The heading angle is stabilized by using the local magnetic field from the magnetometer.

The attitude given by the MTi-G is computed using an EKF. In the prediction step, the gyroscope data are integrated over time to obtain an orientation estimates (by a dead-reckoning technique). The accelerometer calculates attitude estimations by using the gravity vector projection. So, it leads to improve the orientation estimates from the gyroscope. Due to small errors in the gyroscope and transient accelerations (linear accelerations coming from the movement), the attitude estimates suffer from 


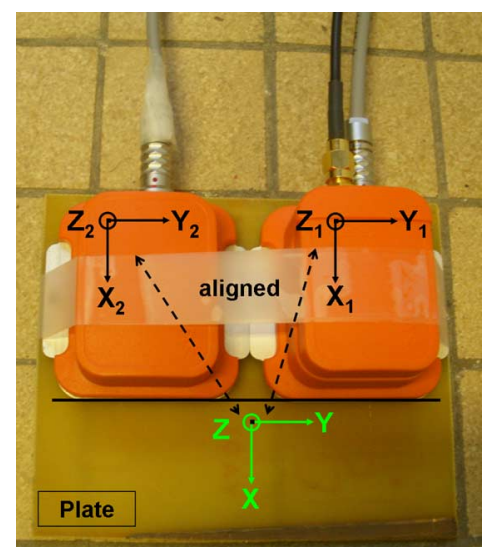

Fig. 5. Whole system. The frame axes of the plate, MTi, and MTi-G are aligned.

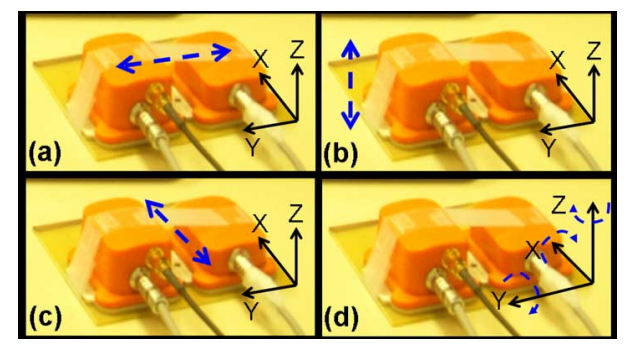

Fig. 6. Motions performed during the evaluation test; the dashed arrow describes the direction of the motion.

some drifts. In the correction step, these drifts are corrected by removing transient accelerations (using a mathematical derivation of velocity measurements coming from GPS receiver) and then the estimation of gravity vector projection is enhanced [21]. The heading is estimated based on GPS and earth's magnetic field measurements.

\section{First Experiment: Improvement of the MTi Shortcomings}

In this first experiment, the performance of the nonlinear filter in estimating the 3-D orientation during a simple motion is compared to the accuracy of the Xsens MT devices. Before starting the motion, the MTi and MTi-G are attached to a plate using an adhesive tape and their frame axes are aligned (see Fig. 5). The experiments were chosen to cover a wide part of the 3-D motions. The subject was asked to perform the four motions outlined in Fig. 6. Thus, the whole system is manipulated to move with up-and-down moving acceleration as follows.

1) Move along the $y$-axis of the plate in the level plane [Fig. 6(a)].

2) Move along the $z$-axis of the plate [Fig. 6(b)].

3 ) Move along the $x$-axis of the plate in the level plane [Fig. 6(c)].

4) Move around $x-, y$-, and $z$-axes of the plate (free motion) [Fig. 6(d)].

The nonlinear filter is fed with calibrated data from the MTi-G to estimate the quaternion describing the orientation in each motion. We chose to express the orientation in Euler angles representation because it is more intuitive than quaternion for the reader. The mathematical transformation between the quaternion and Euler angles is given in [30]. Fig. 7 shows the time

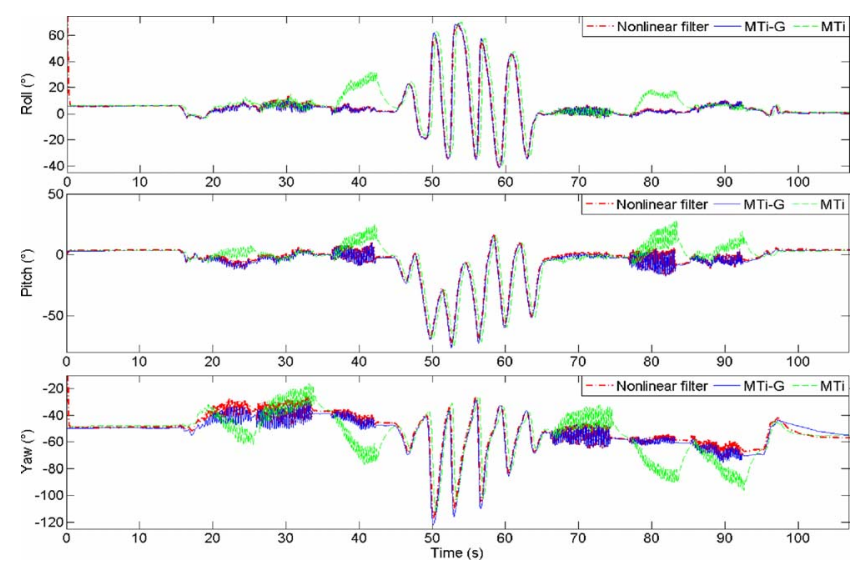

Fig. 7. Attitude estimation with the MTi, MTi-G, and nonlinear filter.

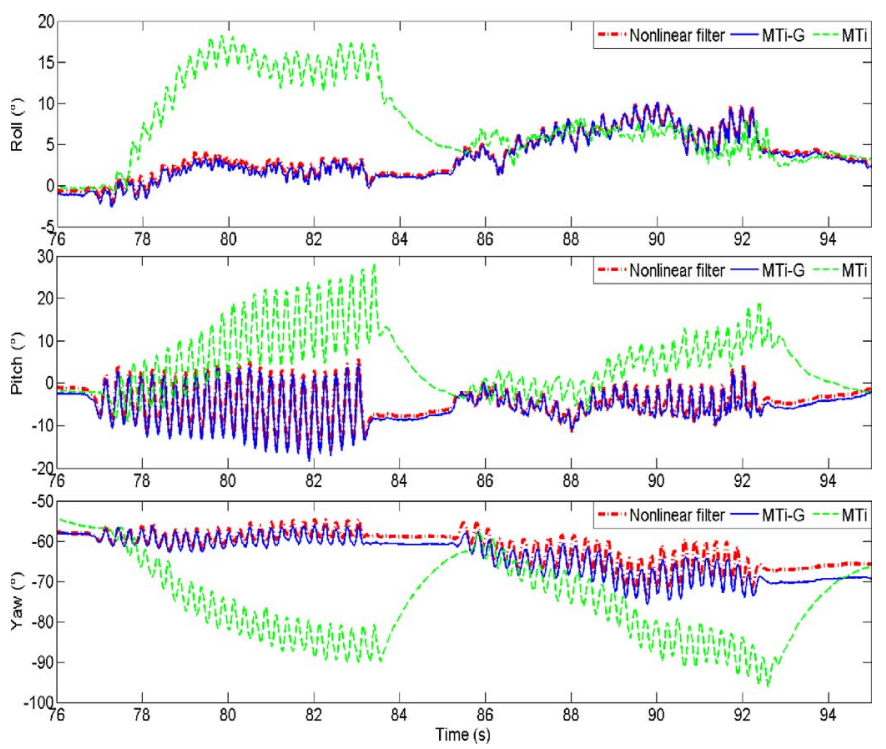

Fig. 8. Zoom of the interval (76-95 s).

history evolution of the Euler angles (roll, pitch and yaw) calculated by the MTi, MTi-G and nonlinear filter. It is important to note that during the intervals (17-34 s), (37-42 s), and (76-95 s), the motion is performed with high linear acceleration (DBA). One of these intervals (76-95 s) is zoomed in Fig. 8 for more clarity. This figure shows the effectiveness of the nonlinear filter in estimating the 3-D orientation during the motion since the differences between the MTi-G and nonlinear filter outputs are small (about $2^{\circ}$ on roll, $2^{\circ}$ on pitch and $3^{\circ}$ on yaw). In conclusion, this experiment shows the drawbacks of the MTi Kalman Filter in estimating orientation and confirms data already published about its shortcoming [31].

\section{Second Experiment: Human Segment Motion Evaluation}

An experimental trial was designed to evaluate the body motion of one subject. The MTi-G is attached to the hand using an adhesive tape (see Fig. 9). Some efforts are carried out to align the axes frame of the MTi-G with those of the hand to represent the ongoing orientation of the subject. To show the nonlinear filter effectiveness, the experiments were chosen to cover a wide part of 3D hand motion. Then, the subject was asked to perform 


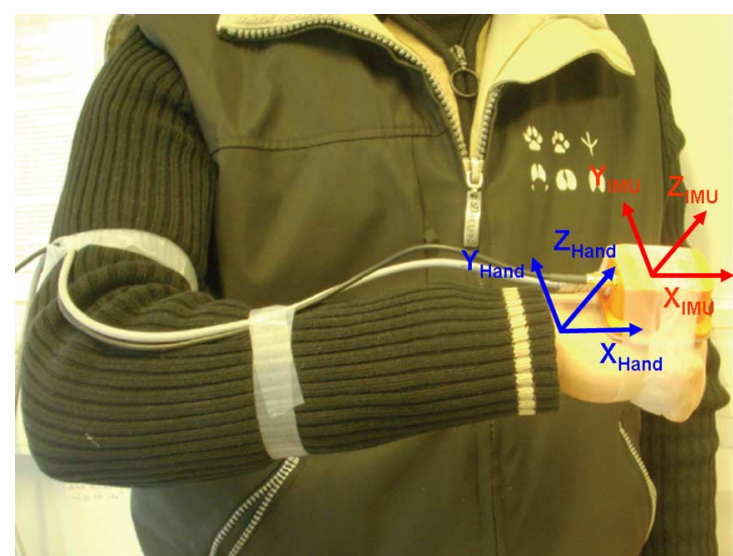

Fig. 9. Subject with the MTi-G attached to the hand. (a)

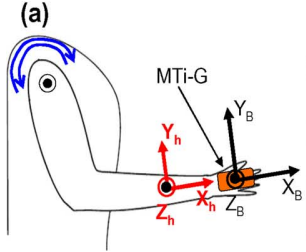

(b)

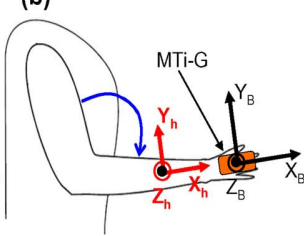

(c)

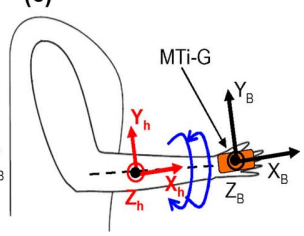

Fig. 10. Exercises performed during the hand motion. (a) Clockwise and anticlockwise shoulder rotation. (b) Elbow extension. (c) Clockwise and anti-clockwise rotation around the dashed line axis defined along the forearm segment.

the three exercises outlined in Fig. 10. The three investigated experiments are carried out with low and high moving acceleration as follows.

1) Clockwise and anti-clockwise shoulder rotation [Fig. 10(a)].

2) Elbow extension [Fig. 10(b)].

3) Clockwise and anti-clockwise rotation around the dashed line axis defined along the forearm segment [Fig. 10(c)].

While the number of subjects used was limited to one, the range of movement over which the technique is evaluated is large and comprehensive. The quaternion errors are shown in Fig. 11 to provide an overview of the overall performance of the nonlinear filter. These errors are computed as the difference between quaternion estimates produced by the nonlinear filter and the MTi-G. The performance level consistency of the complementary filter may be observed in this figure, even in dynamic situations. These estimation errors are very small and the convergence rate is very fast (around $2 \mathrm{~s}$ ). This performance is also proved by using the rmse criterion. So, the orientation estimates of $q$ are accurate within $0.008 \mathrm{rms}$ on $s, 0.0065 \mathrm{rms}$ on $v_{x}$, $0.009 \mathrm{rms}$ on $v_{y}$, and $0.0104 \mathrm{rms}$ on $v_{z}$. It is important to note that, although our approach did not exploit GPS data, it is able to reconstruct the orientation of the hand (given by the MTi-G) with a small error.

\section{E. Third Experiment: Free Movement of Animal}

In this set of experiments, the accuracy of the nonlinear filter is evaluated during the free motion of a domestic animal [29]. Then we chose to carry out tests on a horse. Before starting experiments, the MTi-G is attached to the head of the horse [see

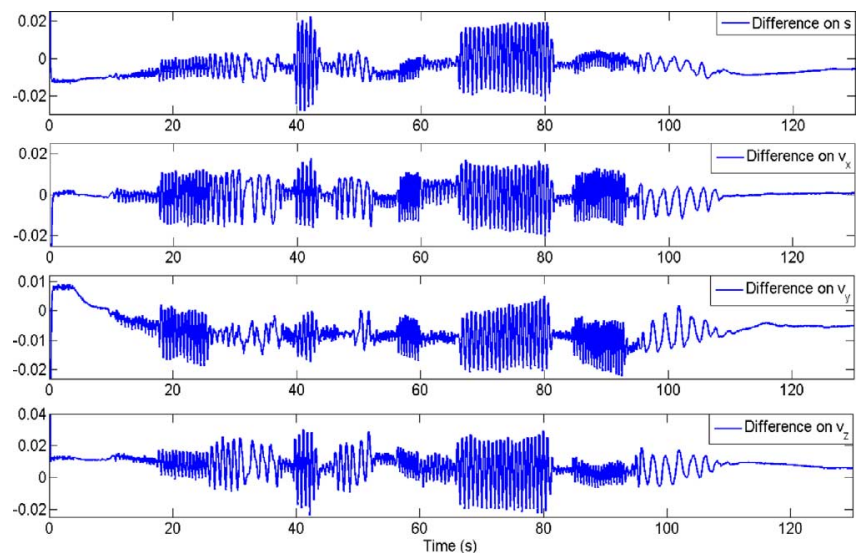

Fig. 11. Differences between quaternion estimates produced by the complementary nonlinear filter and the MTi-G.
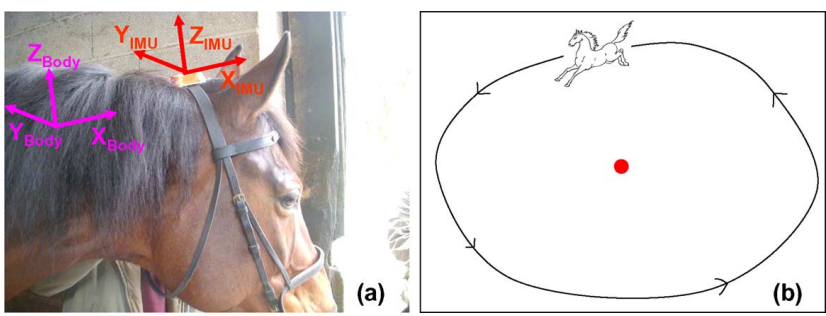

Fig. 12. (a) MTi-G attached to the head of the horse. (b) Schematic diagram of how the horse performed its motion.

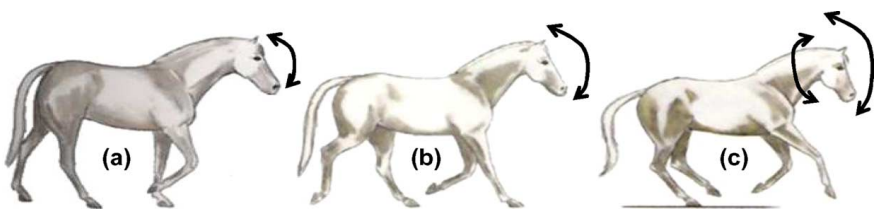

Fig. 13. Gaits of the horse and the movement performed by the head. (a) Walk. (b) Trot. (c) Gallop.

Fig. 12(a)]. The choice of this body limb stems from our biologist colleagues' need to evaluate the head motions and the DBA of the horse during its course. So, this information helps them to study its behavior during the high level competitions.

During the movement, the circular course of the horse (see Fig. 12(b)) is composed of natural gaits such as a walk, trot, and gallop. The experiment is started by a walk where the horse moves its head and neck in a slight up and down motion. During the trot, the horse made small balancing motions with its head. The movement of the horse is finished by a gallop. In this gait, the horse moves its head faster in up/down and right/left motions. The three gaits and head motions of the horse are depicted in Fig. 13.

Fig. 14 plots the evolution of the difference between quaternion estimates produced by the nonlinear filter and the MTi-G. This figure illustrates the performance of the complementary nonlinear filter in estimating the orientation of the horse's head even in dynamic situations (gallop). The orientation estimates of the quaternion $q$ are accurate within $0.0201 \mathrm{rms}$ on $s, 0.0074 \mathrm{rms}$ on $v_{x}, 0.0062 \mathrm{rms}$ on $v_{y}$, and $0.0203 \mathrm{rms}$ on $v_{z}$. Fig. 15 shows the time history evolution of the Euler angles calculated by the nonlinear filter and the MTi-G, plotted on different scales. The 

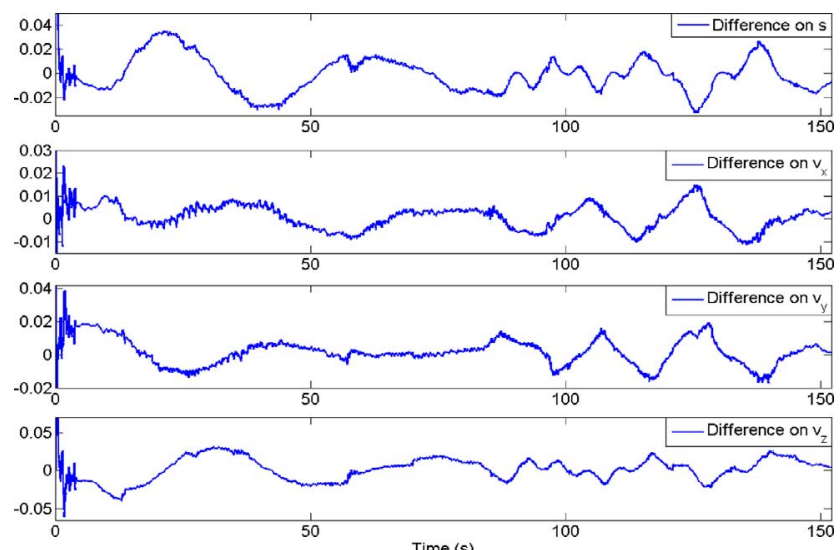

Time (s)

Fig. 14. Differences between quaternion estimates produced by the complementary nonlinear filter and the MTi-G.

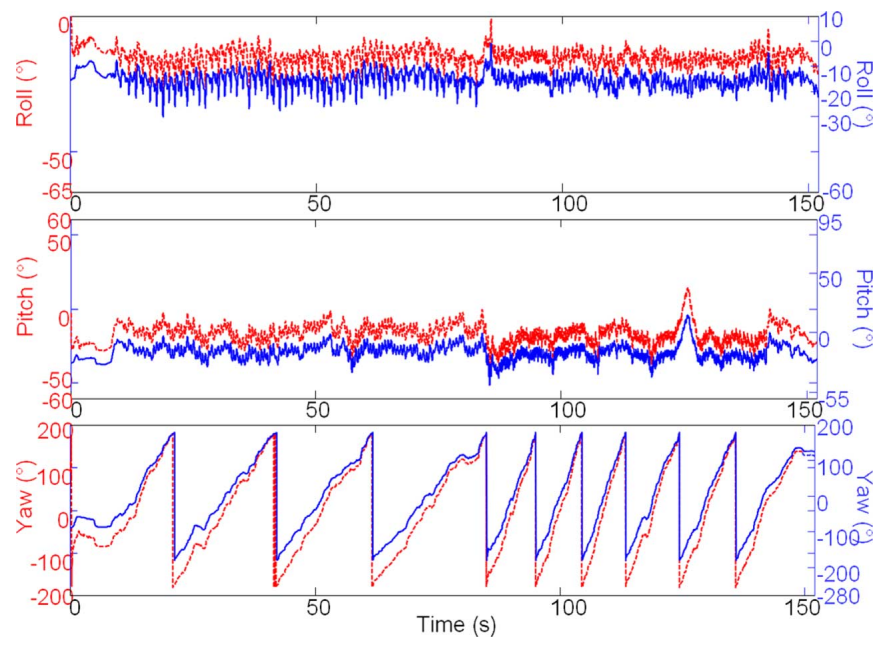

Fig. 15. Euler angles estimates calculated by the nonlinear filter (dashed line) and the MTi-G (solid line).

Euler angles estimation errors are shown in Fig. 16 to provide an overview of the overall performance of the nonlinear filter. These errors are computed as the difference between Euler angles estimates produced by the nonlinear filter and the MTi-G. Note that this difference is always smaller than $3^{\circ}$ on roll, $3^{\circ}$ on pitch and $5^{\circ}$ on yaw, even during fast motions. In static and quasi-static situations (walk and trot), the difference is smaller than $2^{\circ}$. The rms differences between filter and MTi-G outputs during the free movement experiments are within $0.392^{\circ} \mathrm{rms}$ on roll, $0.577^{\circ} \mathrm{rms}$ on pitch, and $2.521^{\circ} \mathrm{rms}$ on yaw. This mismatch is still acceptable in our application and allows implementing the filter in the final prototype.

In our opinion, the differences between the quaternion outputs of the MTi-G Kalman filter and the nonlinear filter are due to different reasons. First, the MTi-G technical documentation [21] indicates that the orientation errors committed by the Xsens Kalman filter in dynamic situations are as follows: pitch/roll: $1^{\circ}$ and yaw: $3^{\circ}$. Second, the internal algorithm of the MTi-G uses GPS measurements such as the velocity to calculate transient accelerations (moving accelerations). This information is used to correct the attitude estimated by the gyroscope. The GPS signal
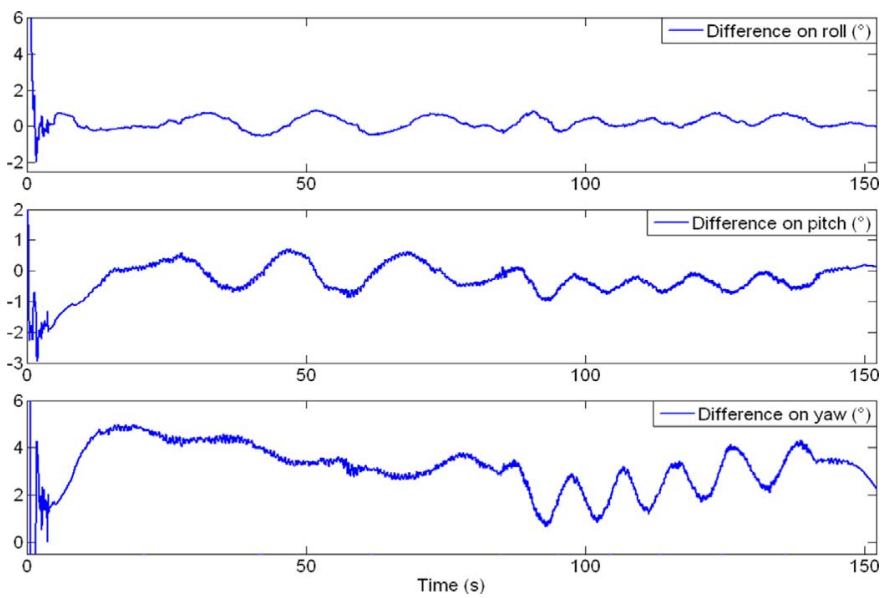

Fig. 16. Differences between Euler angles calculated by the nonlinear filter and the MTi-G.
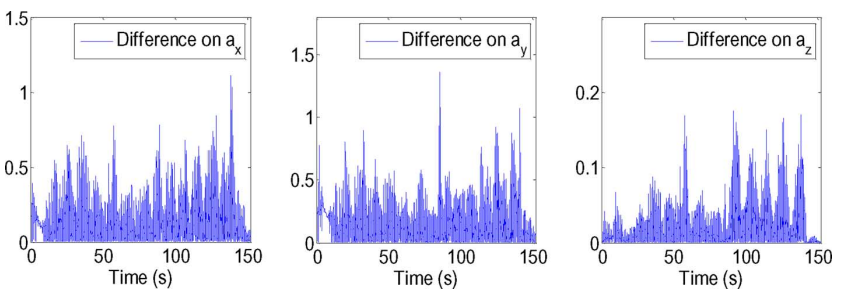

Fig. 17. Differences between the DBA calculated using the complementary nonlinear filter and the MTi-G.

is not always good during the motion, because of the lack of satellites in view, presence of obstructions to line-of-sight to the satellites and atmospheric conditions. Then the transient acceleration calculation is degraded and consequently the precision of the KF is less accurate for short time.

To represent more accurately the ongoing orientation of the subject (the head of the horse or body limb), some efforts are made to align the three axes of the body frame (the subject) with respect to the sensor frame so that the estimated orientation by the filter or by the MTi-G represents the real attitude of the subject. To be sure that the algorithm represents the ongoing orientation of the subject, a manual step can be done after the attitude estimation of $\hat{q}$ by the filter or the MTi-G. This step is given in the Appendix.

The DBA performed by the head of the horse can be determined using the following equation:

$$
\hat{a}=i n v\left(M_{N}^{B}(\hat{q})\right) f-g
$$

where $M_{N}^{B}(\hat{q})$ is the rotation matrix defined in (18), $g$ is the gravity vector, and $f$ is the accelerometer readings.

Fig. 17 plots the evolution of the difference between the DBA estimates produced by the nonlinear filter and the MTi-G. The performance level consistency of the complementary filter may be observed in this figure since the difference is very small. Indeed, the corresponding rms difference is within $0.18 \mathrm{~m} / \mathrm{s}^{2}$ on $a_{x}, 0.19 \mathrm{~m} / \mathrm{s}^{2}$ on $a_{y}$, and $0.03 \mathrm{~m} / \mathrm{s}^{2}$ on $a_{z}$. Such information on the DBA will be necessary in the future to assess energy expenditure of the studied animal species. 


\section{CONCLUSION}

This paper proposes a quaternion-based nonlinear filter for the rigid body motion tracking. The filter was designed with the goal of being able to produce highly accurate orientation and DBA estimates over wide motion range in free-ranging animals (bio-logging). We have proposed a state estimation algorithm that adds to the data from a three-axis accelerometer and a three-axis magnetometer the one provided by a three-axis gyroscope. The main idea is to combine a strap-down system, based on the time integral of angular velocity, with the LMA. The estimated attitude is then exploited to calculate the DBA of the moving body (the animal in our case). The performance of the filter is demonstrated by some simulations and quantitative assessments. Moreover, the efficiency of the proposed approach is shown with a set of experiments on a domestic animal through sensor measurements provided by an Inertial Measurement Unit (MTi-G). The experimental results point out that the algorithm is able to track the accurate orientation and DBA under several motions. Indeed, the rms difference on DBA is within $0.18 \mathrm{~m} / \mathrm{s}^{2}$ on $a_{x}, 0.19 \mathrm{~m} / \mathrm{s}^{2}$ on $a_{y}$, and $0.03 \mathrm{~m} / \mathrm{s}^{2}$ on $a_{z}$.

\section{APPENDIX}

Quaternion: The unit quaternion, denoted by $q$, is expressed as

$$
q=s+v=s+v_{x} i+v_{y} j+v_{z} k \in Q
$$

where $v=v_{x} i+v_{y} j+v_{z} k$ represents the imaginary vector, $s$ is the scalar element, and $Q$ can be written such as

$$
Q=\left\{q^{T} q=1, \quad q=\left[\begin{array}{ll}
s & v^{T}
\end{array}\right]^{T}, \quad s \in \Re, \quad v \in \Re^{3 \times 1}\right\} .
$$

The quaternion product of $q_{a}=\left[\begin{array}{ll}s_{a} & v_{a}^{T}\end{array}\right]^{T}$ and $q_{b}=$ $\left[\begin{array}{ll}s_{b} & v_{b}^{T}\end{array}\right]^{T}$ is defined such as

$$
q_{a} \otimes q_{b}=\left[\begin{array}{cc}
s_{a} & -v_{a}^{T} \\
v_{a} & I_{3 \times 3} s_{a}+\left[v_{a} \times\right]
\end{array}\right]\left[\begin{array}{l}
s_{b} \\
v_{b}
\end{array}\right]
$$

where $I_{3 \times 3}$ is the identity matrix and the operator $\left[v_{a}^{\times}\right]$represents the standard vector cross product which is defined as

$$
\left[v_{a} \times\right]=\left[\begin{array}{ccc}
0 & -v_{a z} & v_{a y} \\
v_{a z} & 0 & -v_{a x} \\
-v_{a y} & v_{a x} & 0
\end{array}\right]
$$

The rotation matrix in term of quaternion can be written as

$$
\begin{aligned}
& M_{N}^{B}(q)= \\
& {\left[\begin{array}{ccc}
2\left(s^{2}+v_{x}^{2}\right)-1 & 2\left(v_{x} v_{y}+s v_{z}\right) & 2\left(v_{x} v_{z}-s v_{y}\right) \\
2\left(v_{x} v_{y}-s v_{z}\right) & 2\left(s^{2}+v_{y}^{2}\right)-1 & 2\left(s v_{x}+v_{y} v_{z}\right) \\
2\left(s v_{y}+v_{x} v_{z}\right) & 2\left(v_{y} v_{z}-s v_{x}\right) & 2\left(s^{2}+v_{z}^{2}\right)-1
\end{array}\right] .}
\end{aligned}
$$

The estimation error on the quaternion is denoted by

$$
E_{q}=\left\|\left(q^{-1} \otimes \hat{q}\right)-1\right\| .
$$

Rigid Body Kinematic Model: The kinematic differential equation, in terms of the unit quaternion, that describes the re- lationship between the rigid body attitude variation and the angular velocity in the body frame is represented by [32]

$$
\dot{q}=\frac{1}{2} q \otimes \omega_{q}
$$

where $\omega_{q}=\left[\begin{array}{ll}0 & \omega^{T}\end{array}\right]^{T}$ is the equivalent update quaternion relative to the angular velocity $\omega \in \Re^{3}$ of the rigid body measured in $B$ and relative to $N . \otimes$ denotes the quaternion multiplication (16).

Sensor Measurement Notations: We give some notations of the sensor measurements.

$$
\begin{array}{lll}
\omega=\left[\begin{array}{lll}
\omega_{x} & \omega_{y} & \omega_{z}
\end{array}\right]^{T} & \text { Real angular velocity. } \\
a=\left[\begin{array}{lll}
a_{x} & a_{y} & a_{z}
\end{array}\right]^{T} & \text { Linear acceleration (DBA). } \\
g=\left[\begin{array}{lll}
g_{x} & g_{y} & g_{z}
\end{array}\right]^{T}= & \text { Gravity vector. } \\
{\left[\begin{array}{lll}
0 & 0 & 9.81
\end{array}\right]^{T}} &
\end{array}
$$

$m=\left[\begin{array}{lll}m_{x} & m_{y} & m_{z}\end{array}\right]^{T} \quad$ Earth's magnetic field .

The theoretical model of the magnetic field nearest to reality is given in [33] and considers a magnetic field vector with a dip angle $\theta=60^{\circ}$ and a norm vector $\|m\|=0.5$ Gauss, so that: $m=\left[\begin{array}{lll}0.5 \cos \left(60^{\circ}\right) & 0 & 0.5 \sin \left(60^{\circ}\right)\end{array}\right]^{T}$.

Usually, the magnetometer error is described by taking into account the effects of external magnetic interferences. These errors can be detected and minimized using an automatic procedure of in-line calibration [34].

$$
b=\left[\begin{array}{lll}
b_{x} & b_{y} & b_{z}
\end{array}\right]^{T}
$$

Bias in the gyroscope which is function of environmental conditions.

Levenberg Marquardt Algorithm Details: The components of $\hat{y}=\left[\begin{array}{llllll}\hat{f}_{x} & \hat{f}_{y} & \hat{f}_{z} & \hat{h}_{x} & \hat{h}_{y} & \hat{h}_{z}\end{array}\right]^{T}$ are calculated by

$$
\begin{aligned}
& \hat{f}=\left[\begin{array}{llll}
0 & \hat{f}_{x} & \hat{f}_{y} & \hat{f}_{z}
\end{array}\right]^{T}=\hat{q}^{-1} \otimes g_{q} \otimes \hat{q} \\
& \hat{h}=\left[\begin{array}{llll}
0 & \hat{h}_{x} & \hat{h}_{y} & \hat{h}_{z}
\end{array}\right]^{T}=\hat{q}^{-1} \otimes m_{q} \otimes \hat{q}
\end{aligned}
$$

where $g_{q}=\left[\begin{array}{llll}0 & 0 & 0 & 9.8\end{array}\right]^{T}$ is the quaternion representation of the gravity vector and $m_{q}=\left[\begin{array}{llll}0 & 0.5 \cos \left(60^{\circ}\right) & 0 & 0.5 \sin \left(60^{\circ}\right)\end{array}\right]^{T}$ is the quaternion representation of the earth's magnetic field.

The Jacobian matrix $X \in \Re^{6 \times 3}$ is defined in [35] as

$$
X=-2\left[\begin{array}{cccccc}
0 & -\hat{f}_{z} & \hat{f}_{y} & 0 & -\hat{h}_{z} & \hat{h}_{y} \\
\hat{f}_{z} & 0 & -\hat{f}_{x} & \hat{h}_{z} & 0 & -\hat{h}_{x} \\
-\hat{f}_{y} & \hat{f}_{x} & 0 & -\hat{h}_{y} & \hat{h}_{x} & 0
\end{array}\right] \text {. }
$$

The gain factor $k$ in (10) is used to tune the balance between measurement noises suppression and response time of the filter. Generally, it combines low bandwidth accelerometer/ magnetometer readings with high bandwidth gyroscope measurements. Notice that the nonlinear filter has a better convergence when $k$ is chosen somewhere between 0.1 and 1 [1].

The gain $K$ takes the following form:

$$
K=k\left[X^{T} X+\lambda I_{3 \times 3}\right]^{-1} X^{T} .
$$




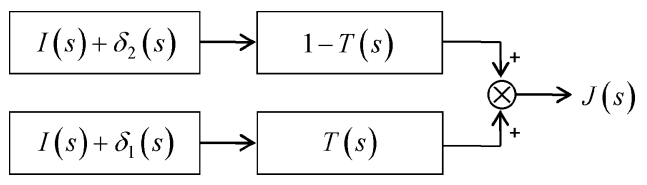

Fig. 18. Complementary filter.

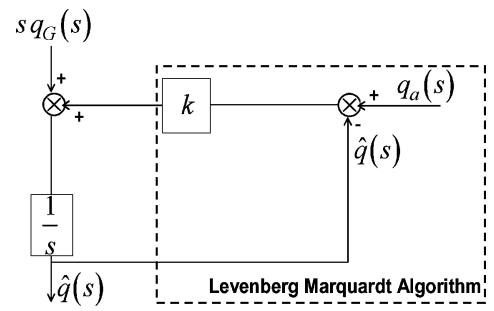

Fig. 19. Transform-domain block diagram (Laplace) of the linearized quaternion estimation filter.

The correction term is computed using the gain $K$ such as

$$
\Delta=\left[\begin{array}{ccccccc}
1 & 0 & 0 & 0 & 0 & 0 & 0 \\
0 & & & & & & \\
0 & & & & K & & \\
0 & & & & & &
\end{array}\right]\left[\begin{array}{c}
1 \\
\delta(\hat{q})
\end{array}\right] .
$$

Complementary Filter Performance: Complementary filtering is a theory that is based upon the use and availability of multiple independent noisy data of the same signal. This technique has been deeply rooted in many papers and books such as [26], [36]. An unknown signal can be estimated using corrupted measurements from one or more sensors whose information naturally stands in distinct and complementary frequency bands [37]. Complementary filtering explores the sensor redundancy to successfully reject measurements disturbances in complementary frequency regions without distorting the signal. If the measurements have complementary spectral characteristics, transfer functions may be chosen in such a way as to minimize estimation error. The general requirement is that one of the transfer functions complements the sum of the others. Thus for measurements of a signal

$$
1-T_{1}(s)-T_{2}(s)-\cdots-T_{n-1}(s)=T_{n}(s)
$$

where $s$ is the time integration Laplace operator.

The simplest complementary filter involves two noise contaminated measurements of a signal. This situation is shown in Fig. 18.

If $\delta_{1}$ is predominantly high-frequency noise and $\delta_{2}$ is low-frequency noise, the two noise sources have complementary spectral characteristics. If $T(s)$ is chosen as a low-pass filter, then $1-T(s)$ is a high-pass filter and both noise signals are attenuated. Since both high and low frequency data are utilized, the filter output will not suffer from any delay in dynamic response due to low-pass filtering.

Indeed, the complementary filtering theory is used in the paper. To study the performance of the nonlinear filter let us show the transform domain block diagram of the linearized quaternion estimation filter. This diagram (see Fig. 19) is obtained from Fig. 1.
The transform of the quaternion from accelerometer and magnetometer readings is $q_{a}(s)$, while $q_{G}(s)$ is the quaternion obtained by integrating gyroscope signals. $s q_{G}(s)$ is the Laplace transform of $\dot{q}_{G}$ [see (5)]. In our case, the accelerometer is noisy and sensed both gravitational acceleration and DBA. The gyroscope suffers from bias. Therefore, $q_{a}(s)$ and $q_{G}(s)$ are both perturbed.

From Fig. 18, the filter transfer function based on accelerometer and magnetometer inputs is given by

$$
F_{1}(s)=\frac{\hat{q}(s)}{q_{a}(s)}=\frac{k s^{-1}}{1+k s^{-1}}=\frac{k}{s+k} .
$$

Equation (27) has the form of a first-order low-pass filter. This proves that the LMA optimization is integrated into the quaternion estimation filter to help obtaining a low-pass filter on $q_{a}(s)$ with respect to the output $\hat{q}(s)$. So, the perturbations due to high-frequency components of accelerometer signal (DBA) are filtered from $q_{a}(s)$.

Similarly, from Fig. 18, the filter transfer function based on gyroscope input is given by

$$
F_{2}(s)=\frac{\hat{q}(s)}{q_{G}(s)}=\frac{1}{1+k s^{-1}}=\frac{s}{s+k} .
$$

Equation (28) has the form of first-order high-pass filter. The gyroscope measurements are high-pass filtered with respect to the output $\hat{q}(s)$. So, the perturbations due to low frequency components of gyroscope signal (the biases) are filtered from $q_{G}(s)$.

Finally, (26) can be verified as

$$
\frac{\hat{q}(s)}{q_{a}(s)}+\frac{\hat{q}(s)}{q_{G}(s)}=\frac{k}{s+k}+\frac{s}{s+k}=1 .
$$

This equation confirms the complementary aspect of the filter. The global transfer function of the filter is written as

$$
\hat{q}(s)=\left(\frac{k}{s+k}\right) q_{a}(s)+\left(\frac{s}{s+k}\right) q_{G}(s) .
$$

One can conclude from (30) that the complementary filter blends low-pass filtering on the signals from the accelerometer-magnetometer pair, and high-pass filtering on the signals from rate gyroscope.

RMSE:

$$
R M S=\sqrt{\frac{1}{T} \sum_{T} x^{2}(t)}
$$

where $T$ is the time interval and $x$ is the computed error.

Arbitrary Alignment: Since the attitude $\hat{q}$ is estimated by the nonlinear filter or the MTi-G, one can calculate the rotation matrix $R_{N}^{B}(\hat{q})$. After that, one considers the coordinate system $C$ of the subject (the body limb or the head of the horse) and the rotation matrix $R_{C}^{B}$ can be measured directly based on the difference between two frames $C$ and $B$. Finally, the 3-D orientation that expresses the ongoing attitude of the subject is given by the rotation matrix $R_{N}^{C}=R_{N}^{B}\left(R_{C}^{B}\right)^{T}$.

Some methods are presented in literature to calculate the rotation matrix $R_{C}^{B}$ that describes the orientation of the subject with respect to the sensor frame attached to it. For example, in the case of the horse, we can apply the method proposed in [9]. In 
the case of human limb motion, we can apply the method proposed in [38].

\section{ACKNOWLEDGMENT}

The authors would like to thank the Ph.D. students of AUTO team (CReSTIC), especially Dr. T. Bouarar and D. Jabri for their help during the horse experiments. The authors would also like to thank the Centre Equestre Reims Université Champagne for allowing the use of the equipments during the experiments.

\section{REFERENCES}

[1] R. Mahony, T. Hamel, and J. M. Pflimlin, "Nonlinear complementary filters on the special orthogonal group," IEEE Trans. Autom. Control, vol. 53, no. 2, pp. 1203-1218, Jun. 2008.

[2] R. Zhu and Z. Zhou, "A small low-cost hybrid orientation system and its error analysis," IEEE Sensors J., vol. 9, no. 3, pp. 223-230, Mar. 2009.

[3] F. Wang and H. Jin, "Design a mini-type marine attitude measurement system for self-propelled model trials," Measurement, vol. 42, no. 6, pp. 954-962, Jul. 2009.

[4] R. Zhu and Z. Y. Zhou, "A real-time articulated human motion tracking using tri-axis inertial/magnetic sensors package," IEEE Trans. Neural Syst. Rehab. Eng., vol. 12, no. 2, pp. 145-162, Jun. 2004.

[5] C. Rutz and G. C. Hays, "New frontiers in biologging science," Biol. Lett., vol. 5, no. 3, pp. 289-292, Mar. 2009.

[6] Y. Ropert-Coudert, M. Beaulieu, N. Hanuise, and A. Kato, "Diving into the world of biologging," Endangered Species Res., vol. 10, pp. 21-27, Apr. 2009.

[7] L. G. Halsey, Y. Handrich, A. Fahlman, A. Schmidt, C. A. Bost, R. L. Holder, A. J. Woakes, and P. J. Butler, "Fine-scale analyses of diving energetics in king penguins Aptenodytes patagonicus: How behaviour affect costs of a foraging dive," Marine Ecol. Progress Series, vol. 344, pp. 299-309, 2007.

[8] G. H. Elkaim, E. B. Decker, G. Oliver, and B. Wright, "Marine Mammal Marker (MAMMARK) dead reckoning sensor for In-Situ environmental monitoring," in Proc. IEEE Position, Location and Navigation Symp., Monterey, CA, Apr. 2006, pp. 976-987.

[9] M. P. Johnson and P. L. Tyack, "A digital acoustic recording tag for measuring the response of wild marine mammals to sound," IEEE J. Ocean. Eng., vol. 28, no. 1, pp. 3-12, Jan. 2003.

[10] S. Watanabe, M. Isawa, A. Kato, Y. Coudert, and Y. Naito, "A new technique for monitoring the behaviour of terrestrial animals; A case study with the domestic cat," Appl. Animal Behaviour Sci., vol. 94, pp. 117-131, 2005.

[11] R. Wilson, E. L. C. Shepard, and N. Liebsch, "Prying into the intimate details of animal lives: Use of a daily diary on animals," Endangered Species Res., vol. 4, pp. 123-137, Jan. 2008.

[12] R. Zhou, D. Sun, Z. Zhou, and D. Wang, "A linear fusion algorithm for attitude determination using low cost MEMS-based sensors," Measurement, vol. 40, no. 3, pp. 322-328, Apr. 2007.

[13] A. M. Sabatini, "Quaternion-based extended Kalman filter for determining orientation by inertial and magnetic sensing," IEEE Trans. Biomed. Eng., vol. 53, no. 7, pp. 1346-1356, Jul. 2006.

[14] J. Thienel and R. M. Sanner, "A coupled nonlinear spacecraft attitude controller and observer with an unknown constant gyro bias and gyro noise," IEEE Trans. Autom. Control, vol. 48, pp. 2011-2015, Nov. 2003.

[15] Analog Devices, Mar. 2009 [Online]. Available: www.analog.com.

[16] Atmel Corporation, Mar. 2009 [Online]. Available: www.atmel.com.

[17] KELLER, Mar. 2009 [Online]. Available: www.keller-druck.ch.

[18] Microchip, Mar. 2009 [Online]. Available: www.microchip.com.

[19] SanDisk Corporation, Mar. 2009 [Online]. Available: http://sandisk.fr.

[20] Wikipedia, Mar. 2009 [Online]. Available: http://en.wikipedia.org/ wiki/Resin.

[21] Xsens Technologies, Mar. 2009 [Online]. Available: www.xsens.com.

[22] M. S. Grewal, L. R. Weill, and A. P. Andrews, Global Positioning Systems, Inertial Navigation, and Integration. New York: Wiley, 2001.

[23] J. B. Kuipers, Quaternion and Rotation Sequences. Princeton, NJ: Princeton Univ., 1999.

[24] S. Beeby, E. Ensell, M. Kraft, and N. White, MEMS Mechanical Sensors. Norwood, MA: Artech House, 2004.
[25] H. Fourati, N. Manamanni, L. Afilal, and Y. Handrich, "Rigid body motions estimation using inertial sensors: Bio-logging application," in Proc. 7thIFAC Symp. Model. Control Biomed. Syst., Alborg, Denmark, Aug. 12-14, 2009.

[26] R. G. Brown and P. Y. C. Hwang, Introduction to Random Signal and Applied Kalman Filterin, 3rd ed. New York: Wiley, 1997.

[27] J. E. Dennis Jr. and R. B. Schnabel, Numerical Methods for Unconstrained Optimization and Nonlinear Equations. Englewood, NJ: Prentice-Hall, 1983.

[28] D. W. Marquardt, "An algorithm for the least-squares estimation of nonlinear parameters," SIAM J. Appl. Math., vol. 11, no. 2, pp. 431-441, 1963.

[29] H. Fourati, N. Manamanni, L. Afilal, and Y. Handrich, "A rigid body attitude estimation for Bio-logging application: A quaternion-based nonlinear filter approach," in Proc. IEEE/RSJ Int. Conf. Intell. Robots Syst., St. Louis, MO, Oct. 2009, pp. 558-563.

[30] W. F. Phillips, C. E. Hailey, and G. A. Gebert, "Review of attitude representations used for aircraft kinematics," J. Aircraft, vol. 38, no. 4, pp. 718-223, Jul.-Aug. 2001

[31] M. A. Brodie, A. Walmsley, and W. Page, "Dynamic accuracy of inertial measurement units during simple pendulum motion," Comput. Methods Biomech. Biomed. Eng., vol. 11, no. 3, pp. 235-242, Jun. 2008b.

[32] M. D. Shuster, "A survey of attitude representations," J. Astronaut. Sci., vol. 41, no. 4, pp. 493-517, Oct.-Dec. 1993.

[33] Astrosurf, Sep. 2009 [Online]. Available: www.astrosurf.com.

[34] D. Roetenberg, H. J. Luinge, C. T. M. Baten, and P. H. Veltink, "Compensation of magnetic disturbances improves inertial and magnetic sensing of human body segment orientation," IEEE Trans. Neural Syst. Rehab. Eng., vol. 13, no. 3, pp. 395-405, Sep. 2005.

[35] H. Fourati, N. Manamanni, L. Afilal, and Y. Handrich, "Nonlinear attitude estimation based on fusion of inertial and magnetic sensors: Bio-logging application," in Proc. 2nd IFAC Int. Conf. Intell. Control Syst. Signal Process., Istanbul, Turkey, Sep. 21-23, 2009.

[36] N. Wiener, Extrapolation, Interpolation and Smoothing of Stationary Time Series. New York: Wiley, 1949

[37] W. Higgins, "A comparison of complementary and Kalman filtering," IEEE Trans. Aerosp. Electron. Syst., vol. AES-11, pp. 321-325, 1975.

[38] K. J. O'Donovan, R. Kamnik, D. T. O'Keeffe, and G. M. Lyons, “A inertial and magnetic sensor based technique for joint angle measurement," J. Biomech., vol. 40, no. 12, pp. 2604-2611, 2007.

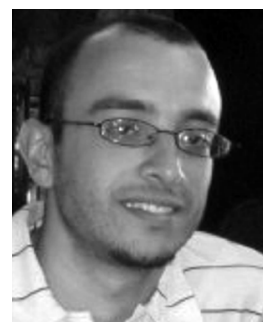

Hassen Fourati (S'09) received the B. Eng. degree in electrical engineering (automatic control) from the National Engineering School of Sfax (ENIS), Sfax, Tunisia, in 2006 . He is currently working toward the Ph.D. degree in electrical engineering at the IPHC-CNRS Laboratory, University of Strasbourg, Strasbourg, France, in collaboration with the CReSTIC laboratory of the University of Reims Champagne Ardenne, France.

His research interests include nonlinear filtering, estimation, and sensor-based navigation with applications in robotics and bio-logging.

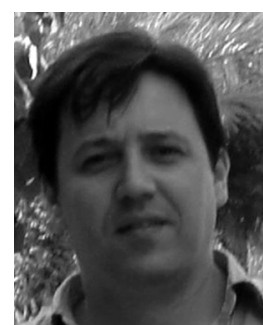

Noureddine Manamanni was born in Annaba, Algeria, in 1967. He received the M.Eng. degree in electrical engineering from the University of Annaba, Annaba, Algeria, in 1990, and the Ph.D. degree in robotics and automation from Université Pierre et Marie Curie-Paris VI, Paris, France, in 1998.

He is currently a Full Professor with the University of Reims Champagne-Ardenne and is affiliated with the CReSTIC Centre de Recherche en Sciences et Techniques de l'Informaion et de la Communication, Reims, France. He is heading the Automatic Control Hybrid Systems, Research Group, CReSTIC. His research interests include nonlinear observer design, estimation, nonlinear control, Lyapunov Stability, hybrid dynamical systems, control of robotic systems, and their application.

Prof. Manamanni is a member of two IFAC Technical Committees and a member of the IEEE Control Systems Society. 


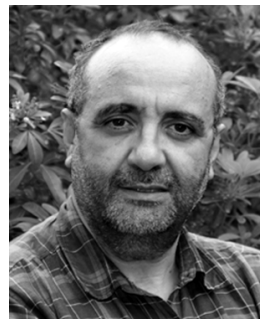

Lissan Afilal received the "Matrise de Physique" degree and the Ph.D. degree in automatic control and computer science from the University of Reims Champagne-Ardenne (URCA), in 1984 and 1990, respectively.

He runs the theme of embedded systems in the Automatic Control and Hybrid System Research Group, CReSTIC Centre de Recherche en Sciences et Technologies de l'Information et de la Communication, Reims, France. His research interests include the automatic biomedical engineering, the control of complex systems, computer engineering and the design of embedded systems. His application areas range from the interoperability of systems on vehicles to bio-logging.

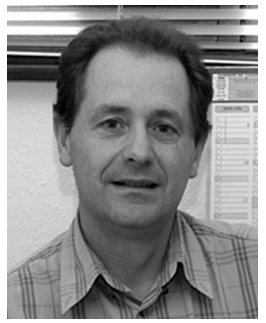

Yves Handrich was born in Paris, France, in 1959. He received the Agregation de physiologi animale in 1984 and the Ph.D. degree in animal ecophysiology from the Université Louis Pasteur, Strasbourg, France, in 1989.

In 1989, he became a Researcher with the Centre National de la Recherche Scientific (CNRS), Reims, France. Since 1989, he has been with CNRS (which became the Institut Pluridisciplinaire Hubert Curien (IPHC-CNRS) in 2007), Strasbourg, France. His research field is focused on the behavioral energetic and ecophysiology of wild animal living in extreme environmental conditions. He is a specialist of fasting, thermoregulation, and diving in marine birds. One key of his approach is the use of the bio-logging technology. 